\title{
A coverage-aware energy replenishment scheme for wireless rechargeable sensor networks
}

\author{
Kuei-Ping Shih ${ }^{*}$ (1) and Chi-Ming Yang
}

\begin{abstract}
Wireless rechargeable sensor networks are becoming crucial and important in recent years for the advancement of wireless energy transmission technology. The previous research showed that not all of sensors can be recharged due to the limitation of energy capacity that mobile chargers can carry. If a sensor playing a critical role in a sensing task cannot function as usual due to exhausted energy, then the sensing task will be interrupted. Therefore, this paper proposes a novel recharging mechanism taking the coverage of sensors into consideration such that mobile chargers can recharge the sensor with a high coverage degree and the network lifetime can be efficiently sustained. The coverage degree of each sensor depends on its contribution to the sensing task, including the coverage and connectivity capabilities. Based on the coverage degree, the sensor with a higher coverage degree will be properly recharged to extend the network lifetime. Simulation results show that the proposed mechanism performs better against the related work in network lifetime.
\end{abstract}

Keywords: Coverage, Energy replenishment, Mobile charger, Sensors, Wireless rechargeable sensor networks

\section{Introduction}

Wireless sensor networks (WSNs) have been widely deployed in numerous fields, such as military surveillance and health care monitoring [1-4]. However, the network lifetime of a WSN is often limited by sensors' energy. As a result, how to extend the network lifetime is becoming the most important issue in WSNs. In addition to power control or power-saving approaches, which are two common approaches to extend network lifetime, in previous research, one of the extending network lifetime schemes is to plan an effective wake-up/sleep schedule for sensors to achieve the purpose of extending the network lifetime [5-8]. In this kind of researches, sensors will automatically judge whether they are redundant $[5,8]$. If the sensor enters in a sleep mode, it can avoid unnecessary power consumption. In $[6,7]$, the goal of this kind of researches is to find a set of sensors to cover the entire area with the minimum number of sensors. However, no matter how to save the

\footnotetext{
*Correspondence: kpshih@mail.tku.edu.tw

Department of Computer Science and Information Engineering, Tamkang University, Tamsui, Taipei, Taiwan
}

energy, the overall network lifetime is still limited by sensors' power.

Recently, wireless energy transmission technology has attracted a lot of attention. The paper [9] uses two identical resonance frequency of magnetic resonance objects to effectively exchange energy. By this way, more sensors can achieve omnidirectional energy transmission. Moreover, this paper also shows a $60-\mathrm{W}$ light bulb at $2 \mathrm{~m}$ far away for wireless lighting and can achieve $40 \%$ energy conversion efficiency. Wireless energy transmission can provide a new way of power supply for WSNs.

The networks in which the sensors can be wireless recharged are called wireless rechargeable sensor networks (WRSNs). There are two most common charging modes used in WRSNs. One is called energy harvesting [10-15], which is to utilize the surrounding environment of energy conversion into electricity to charge sensor nodes, such as wind, water, and solar. Although this way can get energy directly from the surrounding environment, the charging efficiency much depends on the current environment and the locations of the sensors. Therefore, it has a certain degree of difficulty in the reality, because in reality, the change of environment is not

\section{Springer Open}

(c) The Author(s). 2017 Open Access This article is distributed under the terms of the Creative Commons Attribution 4.0 International License (http://creativecommons.org/licenses/by/4.0/), which permits unrestricted use, distribution, and reproduction in any medium, provided you give appropriate credit to the original author(s) and the source, provide a link to the Creative Commons license, and indicate if changes were made. 
predictable, and if the sensor is in a poor place, the sensor cannot get the energy easily.

Therefore, in order to get a more stable way of charging, using mobile chargers equip with wireless energy transfer is the most promising way to provide sustainable energy and has high charging efficiency than energy harvesting. There are many researches in this field [16-26]. In this paper, the sensors are classified into three levels by their contributions in coverage and connectivity to the sensing field. According to the levels, an effective and efficient charging scheme for mobile chargers to recharge sensors is proposed in this paper such that the sensors with high contribution to coverage and connectivity can be charged than those with low contribution in order to prolong the network lifetime.

The remainder of the paper is organized as follows. The literature is reviewed in Section 2. Section 3 introduces some assumptions and background knowledge to help understand the approach of this paper. The proposed scheme and its enhanced version are described in Sections 4 and 5, respectively. Section 6 shows the simulation results, and Section 7 concludes the paper.

\section{Related work}

Using mobile chargers for the sensor nodes to provide electricity is popular in the related literature in WRSNs. The research on planning an effective path for mobile chargers to wireless transfer electricity for sensors according to the remaining energy of sensors is discussed first. In [23], it mainly explored the problem about the energy consumption of mobile charger in moving cost along the path and the replenishment of sensors. The authors want to maximize the profit in sensors' energy replenishment against the moving cost. In addition, the authors also considered the sensor's survival time to avoid the sensor death before the mobile charger can recharge the sensor. In [24], the authors utilized the concept of Named Data Networking (NDN) to effectively get real-time information of the network and the method can also be used in a variety of different network environments. Since the energy information of sensors can be collected effectively, the mobile charger can recharge the sensors in a right time such that the network lifetime can be effectively sustained.

In [18], by considering the time and the location of the sensor issued the recharging request, the problem is formulated as a DMC (on-demand mobile charging) problem. The authors took advantage of the NJNP (NearestJob-Next with Preemption) discipline for mobile charger to select an appropriate sensor to recharge it. In [20], the authors defined the charging throughput maximization problem in heterogeneous networks and transformed it to an orienteering problem in order to plan an efficient charging path for mobile charger. Based on the random energy replenishment, [22] proposed an analytical model to analyze and find an effective way to recharge sensors, where the mobile charger is randomly moved and can find a sensor who is in the sleep mode due to its low energy to recharge. In [21], the authors assumed that the mobile charger has enough energy to recharge sensors. Based on the assumption, the authors studied an optimization problem with the objective of maximizing the ratio of the vacation time of mobile charger over the renewable energy cycle time. Different from the abovementioned studies, [17] explored the recharging problem for the sensors capable to move, not stationary. Therefore, in addition to traditional recharging problem to find an appropriate path for charging, the paper also discussed the rendezvous problem between mobile charger and mobile sensors in order not to miss the recharging of the sensor. However, there are still some literatures that explored how to select appropriate points for the mobile charger, in the charging range, and chose the number of low-energy sensors to charge $[19,25]$.

Although there have been a lot of researches using mobile charger to help sensors get more energy, in these researches, they generally considered the remaining energy of sensors. Figure 1 illustrates an example, where the energies of $s_{1}, s_{2}, s_{3}, s_{4}$, and $s_{5}$ are below the energy threshold and need to be recharged. If the energy replenishment strategy only considered the remaining energy of sensors, the recharging sequence or path is $s_{1} \rightarrow s_{2} \rightarrow$ $s_{3} \rightarrow s_{5} \rightarrow s_{4}$. This kind of strategies did not take into account the importance of sensors in the task. However, the most important things in wireless sensor networks are the coverage of the sensing field and the connection among sensor nodes.

Therefore, not only to consider the remaining energy of sensors but also to consider the coverage issue to ensure the quality of surveillance of the entire sensing field play an important role in wireless sensor networks. The sensors needed to be recharged are the ones which play an important role in the wireless sensor networks. Take Fig. 2 as an example, where the remaining energies of $s_{1}, s_{2}, s_{3}$, and $s_{4}$ are below a threshold and need to be recharged. Moreover, the sensors with the sensing ranges of the same color, either blue- or green-colored circles, means that these sensors can fully cover the sensing range of the red-colored sensor, whose remaining energy is below the threshold. The sensors with the same colored sensing range are grouped into a cover set of the corresponding red-colored sensor. The detailed definition of cover set will be defined later [27]. For example, $s_{2}$ only has one cover set, which is blue colored, and $s_{4}$ has two cover sets, which are blue and green colored. If only the remaining energy is considered, since $s_{2}$ and $s_{4}$ have the same remaining energy, the charging sequence is either $s_{1} \rightarrow$ $s_{3} \rightarrow s_{2} \rightarrow s_{4}$ or $s_{1} \rightarrow s_{3} \rightarrow s_{4} \rightarrow s_{2}$. However, if the 


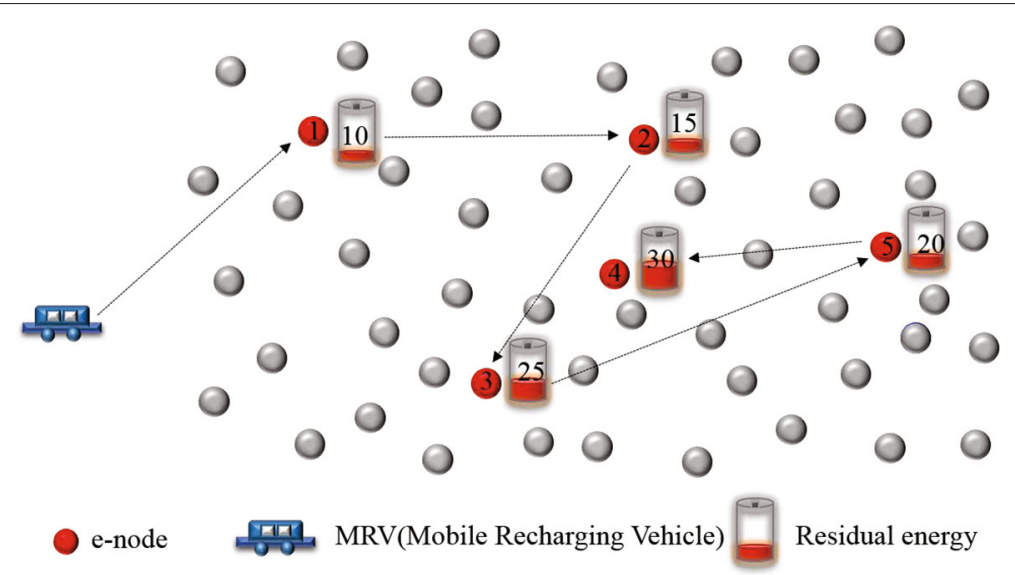

Fig. 1 Illustration of the problem only considering remaining energy of sensors

coverage issue is considered, the priority to recharge $s_{2}$ is higher than that to recharge $s_{4}$. It is because if $s_{4}$ is out of energy, the sets of sensors marked by either blue color or green color can help cover the sensing range of $s_{4}$. However, if $s_{2}$ is out of energy, only one set of sensors marked by blue color can help cover the sensing range of $s_{2}$. Consequently, $s_{2}$ should have a higher priority to be recharged than $s_{4}$ in order not to result in a hole.

As a result, it is important to consider the remaining energy of sensors in conjunction with the importance in coverage and connectivity issues of sensors such that the sensors who really need to be recharged can be recharged. Since the energy carried by the mobile charger is limited, it is not possible to recharge all sensors. When some sensors are inevitable to be sacrificed, the priority of sensors to be recharged turns to be much important.

\section{Preliminaries}

This paper utilizes mobile chargers to recharge sensors with low remaining energy. Not only the remaining energy of a sensor is considered but also the contributions in terms of coverage and connectivity of the sensor are also taken into account while recharging such that the network lifetime can really be extended.

\subsection{Network model and assumptions}

It is assumed that the goal of the sensor network is to make sure the sensing field is area covered, which means that every point in the sensing field should be covered by at least one sensor. It is also known as one covered [28]. The entire network is illustrated in Fig. 3, where sensors are randomly deployed in the sensing field and the sensing field is assumed to be at least one covered. Let $S$ denote

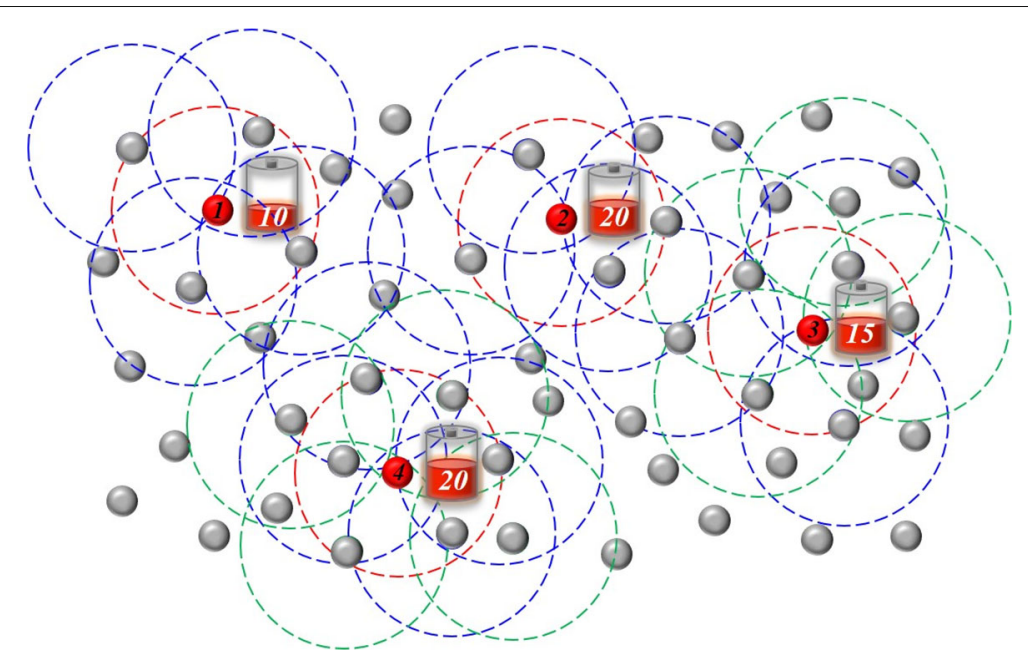

Fig. 2 Illustration of the problem considering coverage and remaining energy of sensors 


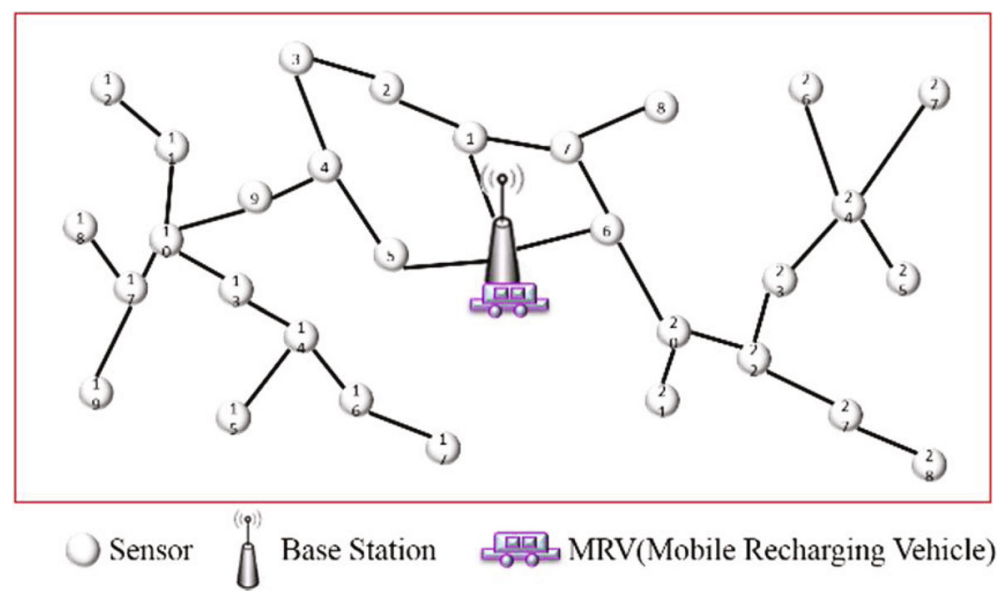

Fig. 3 Network model

the set of sensors. That is, $S=\left\{s_{i}, i=1,2, \cdots\right\}$. Sensors are assumed to be stationary and homogeneous. Sensors have the same capabilities, such as the same sensing range and the same communication range. The sensing range and the communication range of a sensor are denoted $r_{s}$ and $r_{c}$, respectively. The sensing area and the communication area of a sensor are circles centered at the sensor. Any point within the sensing range of a sensor can be detected by the sensor. In the meanwhile, any sensor within the communication range of another sensor can communicate with each other. The sensing area covered by $s_{i}$ is denoted as $\mathcal{C}\left(s_{i}\right)$. On the other hand, let $N\left(s_{i}\right)$ be the set of neighbors of $s_{i}$, which is defined as $N\left(s_{i}\right)=$ $\left\{s_{j} \in S \mid \operatorname{dist}\left(s_{j}, s_{i}\right) \leq r_{c}\right\}$, where $\operatorname{dist}(x, y)$ is the Euclidean distance between $x$ and $y$.

To simplify the discussion, we also assume that $r_{c} \geq 2 r_{s}$. The assumption can ensure the connectivity of sensors when full sensing coverage is preserved. On the other hand, we also assume that a sensor is location-aware. The location of a sensor can be obtained either by GPS or by many existing localization schemes [29, 30]. In addition, the base station has the location information of the sensors.

In addition, in this paper, time is divided into rounds, and each round will be divided into an initial phase and a working phase, as illustrated in Fig. 4. During the initial phase, each sensor sends back the sensed data and its remaining energy to the base station in a multi-hop fashion. The base station starts to plan a charging tour for the mobile charger while receiving the information from the sensors. During the working phase, the mobile charger will recharge the sensors according to the charging tour planed by the base station in the initial phase. The duration that a mobile charger moves from the base station and returns back to the base station is termed a cycle. It is worth mentioning that a working phase may contain more than one cycle, depending on the time spends in a charging tour.

Regarding the mobile charger, it is required that a charging tour in a cycle should start and end at the base station, which is to ensure the mobile charger can carry enough energy at the base station to continuously recharge sensors. The moving speed of the mobile charger is fixed, and the maximum energy a mobile charger can carry is also fixed.

\subsection{Coverage and energy critical node}

In this paper, the energy critical sensor is termed an $e$-node. When an e-node appeared, the mobile charger will be used to recharge this e-node. However, only

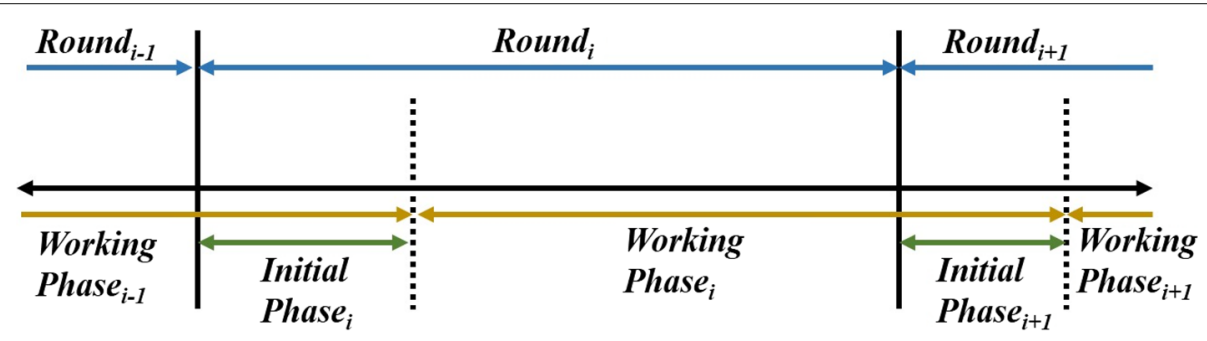

Fig. 4 The operation model in terms of timeline 
considering this condition is not enough. The contribution of sensors to the sensing field needs to be considered as well. The paper assumes that the transmission range is larger than two times of the sensing range. As a result, as long as the network is fully covered without a hole, the network is connected. Thus, the only thing to be considered is the coverage issue in the network. Therefore, the sensor which may lead to a coverage hole is called a coverage-critical node and is denoted c-node. In this paper, the concept of cover set of a sensor is used to represent the contribution level of the sensor. The concept of cover set was first proposed in [7,27]. As a result, the number of cover sets of a sensor can be used to decide whether the sensor is potential to be a c-node or not.

The formal definition of a cover set is given as follows. A cover set of a sensor, say $s_{i}$, is the set of sensors whose sensing areas can completely cover $\mathcal{C}\left(s_{i}\right)$. A cover set of $s_{i}$ is denoted as $C V\left(s_{i}\right)$ and is formally defined in Definition 1.

Definition 1 (Cover set (CV)) $C V\left(s_{i}\right)$ is a set of sensors satisfying the following requirements.

- $\cup_{s_{j} \in C V\left(s_{i}\right)} \mathcal{C}\left(s_{j}\right) \supseteq \mathcal{C}\left(s_{i}\right)$,

- for any $s_{j} \in C V\left(s_{i}\right), \mathcal{C}\left(s_{j}\right) \not \subset \cup_{s_{k} \in C V\left(s_{i}\right), k \neq j} \mathcal{C}\left(s_{k}\right)$.

By Definition 1, we can easily derive the following lemma.

Lemma 1 If $s_{j} \in C V\left(s_{i}\right), s_{j} \in N\left(s_{i}\right)$.

Proof Since $s_{j}$ belongs to $\mathrm{CV}\left(s_{i}\right), \mathcal{C}\left(s_{j}\right) \cap \mathcal{C}\left(s_{i}\right) \neq \emptyset$. It implies that $\operatorname{dist}\left(s_{i}, s_{j}\right) \leq 2 r_{s}$. Moreover, since $r_{c} \geq 2 r_{s}$, $\operatorname{dist}\left(s_{i}, s_{j}\right) \leq r_{c}$. Therefore, $s_{j} \in N\left(s_{i}\right)$.

The concept of the cover set of $s_{i}$ is illustrated in Fig. 5, in which four cover sets, respectively denoted by blue, yellow, purple, and green colors, can be found. Therefore, if the number of cover sets of a sensor is few, the sensor has a high potential to lead to a coverage hole. As a result, the sensor is possible to be a c-node.

\section{Priority-based Energy Replenishment Scheme (PERS)}

This paper presents two different schemes to recharge sensors. The first scheme, named PERS (Priority-based Energy Replenishment Scheme), calculates the weight of each sensor. The remaining energy and number of cover sets of a sensor are taken into account while designing the weight. According to the weight, we divide sensors into three kinds of priorities to recharge the sensors. The higher the priority of a sensor is, the sooner the

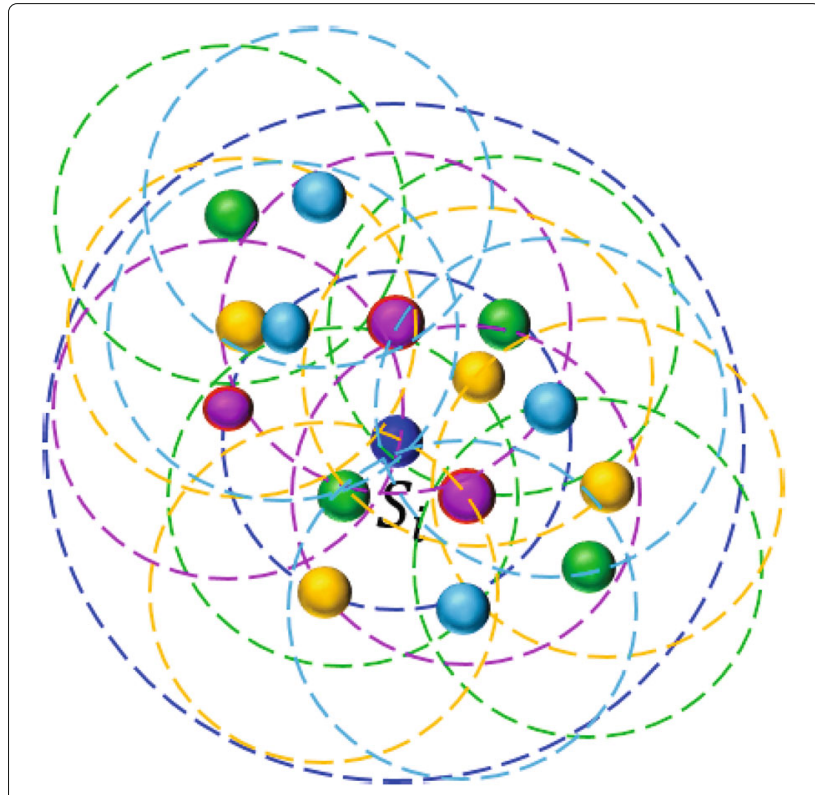

Fig. 5 Illustration of cover sets of $s_{i}$

sensor is charged by the mobile charger. Only when the mobile charger has more energy the sensors with the next priority are considered to be recharged. PERS can recharge the coverage and energy critical sensors with high priority and effectively alleviate the possibility to cause a coverage hole. However, this scheme may result in a long charging tour and consume much power of mobile charger while walking along the path. To avoid this problem, we propose another scheme, named e-PERS (Enhanced Priority-based Energy Replenishment Scheme), where we use a $k$-means clustering algorithm to divide the sensors into $k$ clusters in which the sensors belonging to a cluster are closer than to the other clusters.

PERS mainly consists of the following three steps. First, we design the weight of a sensor. By the weight, sensors are divided into three different priorities, red, yellow, and green priorities. Based on the different priorities, the charging tour for recharging is scheduled.

\subsection{Weight value design}

The remaining energy of a sensor is considered to be a very important factor in wireless rechargeable sensor networks. However, only considering the remaining energy of a sensor is not enough because it is easy to lead to a coverage hole and the sensing task is going to be terminated. Therefore, this paper also takes the coverage critical sensors into account to postpone the occurrence of a coverage hole.

Consequently, a weight is designed for a sensor by combining the remaining energy and coverage importance of 
the sensor. Let $w_{i}$ denote the weight of $s_{i}$ and be calculated by Eq. (1).

$$
w_{i}=\frac{1}{A_{i}^{\mathrm{CV}}+1} \times \frac{B-B_{i}^{\text {res }}}{B},
$$

where $A_{i}^{\mathrm{CV}}$ is the total number of cover sets of $s_{i}, B$ is the maximum battery capacity of a sensor, and $B_{i}^{\text {res }}$ is the remaining energy of $s_{i}$. $A_{i}^{\mathrm{CV}}$ means that when $s_{i}$ is going to exhaust its energy, its sensing range can be covered by $A_{i}^{\mathrm{CV}}$ sets of sensors. The smaller $A_{i}^{\mathrm{CV}}$ is, the sparser $s_{i}$ is covered. Therefore, smaller $A_{i}^{\mathrm{CV}}$ is prone to have a coverage hole at $s_{i}$. On the contrary, the smaller $B_{i}^{\text {res }}$ is, the more possibility $s_{i}$ is going to exhaust its energy soon. The notations and their corresponding definitions are summarized in Table 1.

By Eq. (1), $\frac{1}{A_{i}^{\mathrm{CV}}+1}$ can differentiate among sensors prone to result in a coverage hole and $\frac{B-B_{i}^{\text {res }}}{B}$ can find out the sensors that exhaust most energy. Consequently, a sensor with a larger weight implies that the sensor should have a higher priority to be recharged. As a result, Eq. (1) can differentiate sensors which are prone to have a coverage hole and their remaining energies are also prone to be exhausted. Figure 6 shows an example, and the weights of sensors are respectively shown in Fig. 7, where $B$ is assumed to be $100 \mathrm{~J}$.

\subsection{Classification of sensors}

According to the weights of sensors, sensors are classified into red, yellow, and green groups with different priorities. The red group of sensors has the highest priority and should be recharged first. The sensors in the red group mean that these sensors are going to exhaust their energy in this round and these sensors are also prone to have coverage holes if they run out of energy. The yellow group of sensors has the second priority and can be recharged after the red group of sensors. The sensors in the yellow group mean that these sensors can live more than one round and are also not quite prone to have coverage holes than those in the red group. The least priority is given to the sensors in the green group. Similarly, the sensors in the green group mean that these sensors can live more than two

Table 1 Notations

\begin{tabular}{ll}
\hline Notation & Definition \\
\hline$A_{i}^{\mathrm{CV}}$ & Total number of cover sets of $s_{i}$ \\
$B$ & The maximum battery capacity of a sensor \\
$B_{i}^{\text {res }}$ & The remaining energy of $s_{i}$ \\
AVGCV $^{\text {The average number of cover sets of all sensors }}$ \\
$\theta$ & The amount of energy a sensor consumed in a round \\
$B_{i}^{\mathrm{RE}}$ & The amount energy of a mobile charger replenished to $s_{i}$ \\
$C_{(i, j)}^{\text {run }}$ & The energy consumed by a mobile charger moving from $s_{i}$ to $s_{j}$
\end{tabular}

rounds and are least prone to have coverage holes than those in the other two groups. Equations (2), (3), and (4) are used to classify the sensors into red, green, and yellow groups, respectively.

$$
\begin{aligned}
& w_{i} \geq \frac{1}{\left\lfloor\mathrm{AVG}_{\mathrm{CV}}\right\rfloor+1} \times \frac{B-\theta}{B} \\
& w_{i} \leq \frac{1}{\lceil\mathrm{AVG} \mathrm{CV}\rceil+1} \times \frac{B-2 \theta}{B} \\
& \frac{1}{\left\lceil\mathrm{AVG}_{\mathrm{CV}}\right\rceil+1} \times \frac{B-2 \theta}{B}<w_{i}<\frac{1}{\left\lfloor\mathrm{AVG}_{\mathrm{CV}}\right\rfloor+1} \times \frac{B-\theta}{B}
\end{aligned}
$$

Equations (2), (3), and (4) are derived from Eq. (1). We use an average number of cover sets of all sensors to replace $A_{i}^{\mathrm{CV}}$ in Eq. (1), which means that, if the number of cover sets of a sensor is smaller than the average, the neighbors of the sensor is few and the sensor is deemed to be prone to have a coverage hole. On the other hand, let $\theta$ be the amount of energy a sensor consumed in a round. As mentioned above, the sensors in the red group is going to exhaust their energy in one round. Therefore, $B_{i}^{\text {res }}$ in Eq. (1) is replaced by $\theta$ in Eq. (2). Similarly, regarding the threshold for the green group, $B_{i}^{\text {res }}$ in Eq. (1) is replaced by $2 \theta$ in Eq. (3).

The result of classification for the example shown in Fig. 6 is illustrated in Fig. 8, where $\theta$ is assumed to be $30 \mathrm{~J}$. The two thresholds are 0.35 and 0.133 . That is, the sensors whose weights are larger than or equal to 0.35 are classified into the red group. The sensors whose weights are smaller than or equal to 0.133 are classified into the green group. Otherwise, the sensors are classified into the yellow group.

\subsection{Charging tour planning}

The last stage of PERS is the charging tour planning stage. The charging tour planning is based on the different priorities and the weights of sensors. The strategy in planning the charging tour for the sensors in the red group is different from those for the sensors in the yellow and green groups. In designing the charging tour for the sensors in the red group, we consider the sensors one by one from the highest weight to a lower one. We use a Hamiltonian cycle algorithm to plan the charging tour. The Hamiltonian cycle algorithm has been well studied in the fields of algorithm or graph theory [31] and is not the focus of the paper. Regarding a sensor, if the energy of the mobile charger is not enough to recharge the sensor, the sensor is ignored in this cycle and the charging tour is finished. The sensor will be considered in the next cycle.

The strategy used in the yellow group is a slightly different from the one used in the red group. Since the sensors in the yellow group are not so urgent to be recharged 


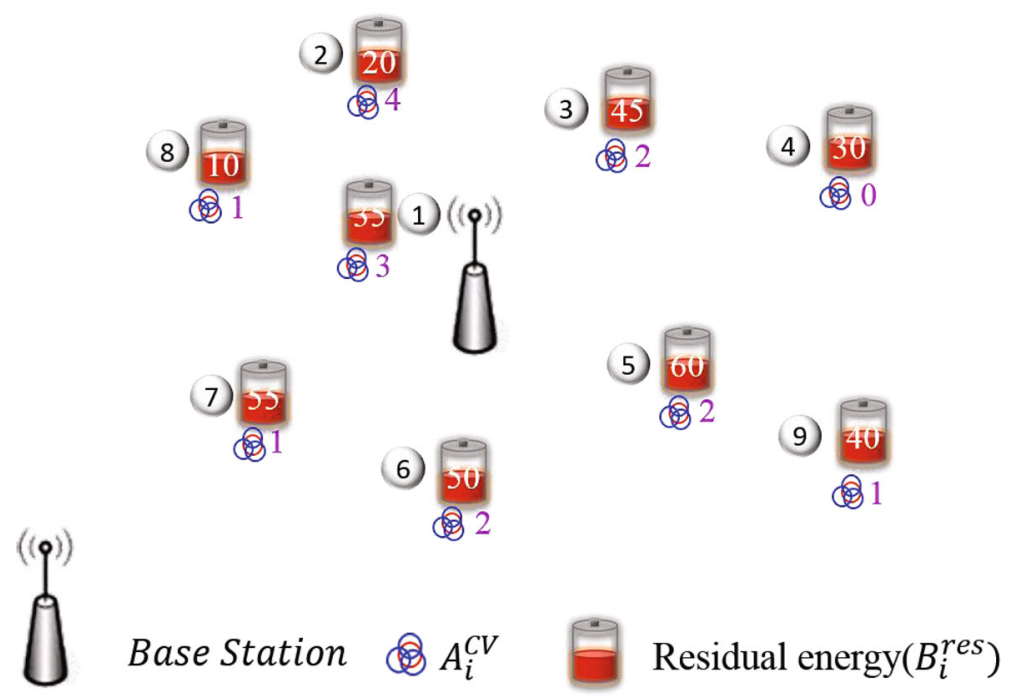

Fig. 6 An example to illustrate the design of the weights of sensors

in this round, we can choose a sensor to be ignored in this cycle such that the resultant charging tour is short or the mobile charger can recharge more sensors. Therefore, when the mobile charger can not recharge a sensor, we will use Eq. (5) to select a sensor to ignore in this cycle, where $C_{(i, j)}^{\text {run }}$ is the energy consumption of the mobile charger in moving from $s_{i}$ to $s_{j}$ and $B_{i}^{\mathrm{RE}}$ is the amount of energy the mobile charger uses to recharge $s_{i}$. This idea is similar to a greedy rule that wants to find a short charging tour in the yellow group but the sensors with higher weights can also have a high priority to be included in the charging tour. Figure 9 is an illustration of the charging tour planning of the sensors in the yellow group. Suppose the order of the weights of the sensors in the yellow group is $w_{8}>w_{9}>w_{7}>w_{3}>w_{6}>w_{1}>w_{2}$. Firstly, we consider $s_{8}$ and then $s_{9}$. Suppose the mobile charger can recharge the two sensors and return to the base station. We then consider the next sensor, $s_{7}$. Suppose the mobile charger has no enough energy to recharge $s_{7}$. We use Eq. (5) to select a sensor to ignore in this cycle. By Eq. (5), $s_{9}$ is ignored in this cycle. After $s_{9}$ is removed, we can get a better charge efficiency $\left(s_{3} \rightarrow s_{8} \rightarrow s_{7}\right)$. If we do not take $s_{9}$ out, we can only charge two sensors $\left(s_{8}\right.$ and $\left.s_{9}\right)$.

$$
\frac{B_{i}^{\mathrm{RE}}}{C_{(i, j)}^{\mathrm{run}}}
$$

The strategy in planning the charging tour for the sensors in the green group is the same with that for the sensors in the yellow group.

Since it is inevitable that the mobile charger needs to consume energy while moving along the charging tour, the energy a mobile charger used to recharge sensors is counted after the energy consumed in moving along the charging tour is deducted. If the mobile charger has enough energy to recharge the sensors, the mobile charger will charge the sensors to their maximum capacity. Otherwise, the mobile charger will charge the sensors scheduled in the same cycle to at least $2 \theta$ to ensure these sensors can live at least two rounds and will not fall into the red group soon. After that, if the mobile charger still has more energy, the energy will evenly recharge to these sensors.

\section{Enhanced Priority-based Energy Replenishment Scheme (e-PERS)}

PERS may cause the mobile charger to move inefficiently and redundantly and result in a long charging tour. Therefore, an enhanced version, named enhanced PERS (e-PERS), is also investigated in this paper. e-PERS consists of five steps. The first two steps in e-PERS are the same with the first two steps in PERS. Afterwards, we use the $k$-means clustering algorithm to cluster sensors. The sensors located within the same cluster are in the vicinity of each other.

\begin{tabular}{|c|c|c|c|c|c|c|c|c|}
\hline$W_{4}$ & $W_{8}$ & $W_{9}$ & $W_{7}$ & $W_{3}$ & $W_{6}$ & $W_{1}$ & $W_{2}$ & $W_{5}$ \\
\hline 0.7 & 0.45 & 0.3 & 0.225 & 0.183 & 0.167 & 0.163 & 0.16 & 0.133 \\
\hline
\end{tabular}

Fig. 7 The weights of sensors in the example showed in Fig. 6 


\begin{tabular}{|c|c|c|c|c|c|c|c|c|}
\hline$W_{4}$ & $W_{8}$ & $W_{9}$ & $W_{7}$ & $W_{3}$ & $W_{6}$ & $W_{1}$ & $W_{2}$ & $W_{5}$ \\
\hline 0.7 & 0.45 & 0.3 & 0.225 & 0.183 & 0.167 & 0.163 & 0.16 & 0.133 \\
\hline
\end{tabular}

Fig. 8 The result of classification for the example showed in Fig. 6

After clustering, it is possible that the numbers of sensors in different clusters are imbalanced. The numbers of sensors in different groups in a cluster are also unevenly distributed. Take Fig. 10 as an example, where $k=2$. There are six sensors in the blue-colored cluster and three sensors in the yellow-colored cluster. To be fair, let the cluster with the minimum number of sensors be a base cluster. All the other clusters with more sensors can only take the same number of sensors with the number of sensors in the base cluster into account. In this example, the yellow-colored cluster is the base cluster and the number of sensors within it is 3 . Therefore, we take the three sensors with the highest weights into account for other clusters.

As a result, the second step is to determine which cluster should be considered to recharge sensors first. The easiest way is to select the cluster with the maximum summation of the weights of sensors within the cluster. In the example, the summations of weights of sensors in the blue-colored and the yellow-colored clusters are 1.133 and 0.858 , respectively. Hence, we choose the yellow-colored cluster to recharge first. Finally, the last step in e-PERS is the same as the last step in PERS, that is, to plan a charging tour in the cluster determined in the last step.
It is worth mentioning that $k$ can be determined based on the number of cycles in a round or the number of mobile chargers.

\section{Simulation results}

This section shows the simulation results of the comparisons of PERS and e-PERS with GA proposed in [23] in terms of network lifetime and the remaining energy of sensors. The network lifetime is defined as the duration from the start of the simulation until the time the first coverage hole occurs. The sensing field is $300 \mathrm{~m} \times 300 \mathrm{~m}$. There are 80 sensors randomly deployed in the sensing field. Every sensor is equipped with a maximum power of $500 \mathrm{~J}$. There was a mobile charger in the sensing field, and the velocity of the mobile charger is $5 \mathrm{~m} / \mathrm{s}$. The energy consumed in movement by the mobile charger is $10 \mathrm{~J}$ per second. The recharging rate to a sensor is $5 \mathrm{~J}$ per second. The base station is located in the center of the sensing field. The simulation parameters are shown in Table 2.

In Fig. 11, the method, GA proposed in [23], results in a hole at the 15436th second because the GA method only considers the remaining energy of sensors. The coverage issue is ignored by this method, which leads to the sensors which are prone to have coverage holes running

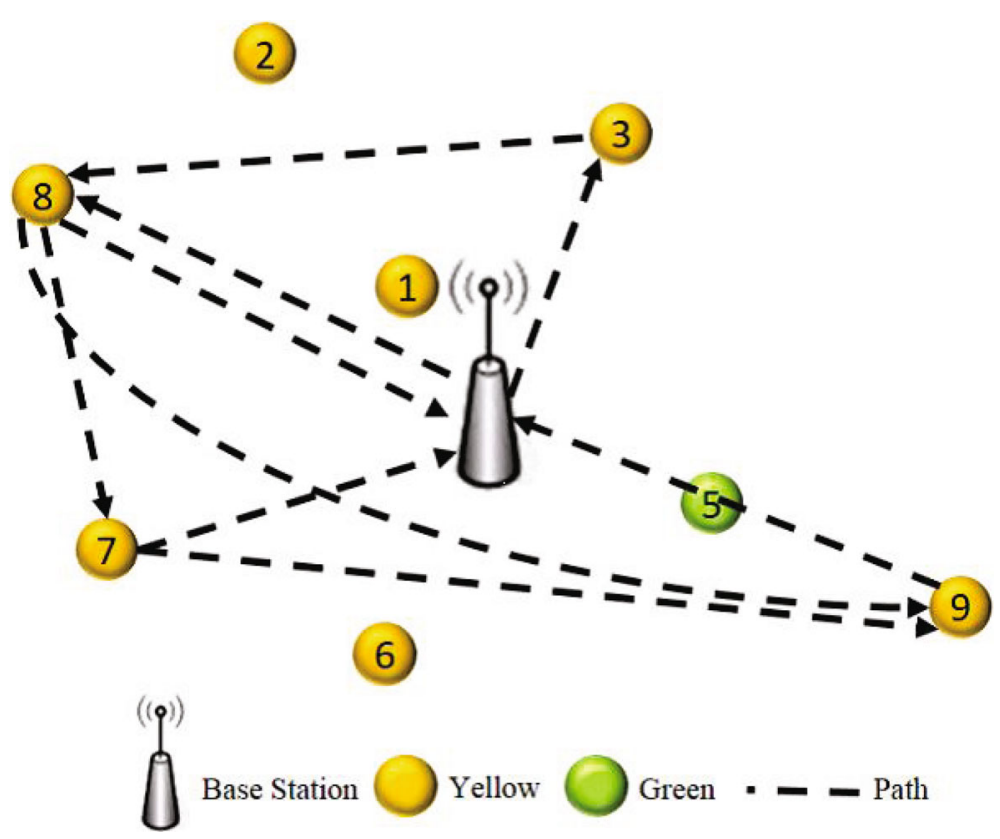

Fig. 9 The charging tour planning of the sensors in the yellow group 


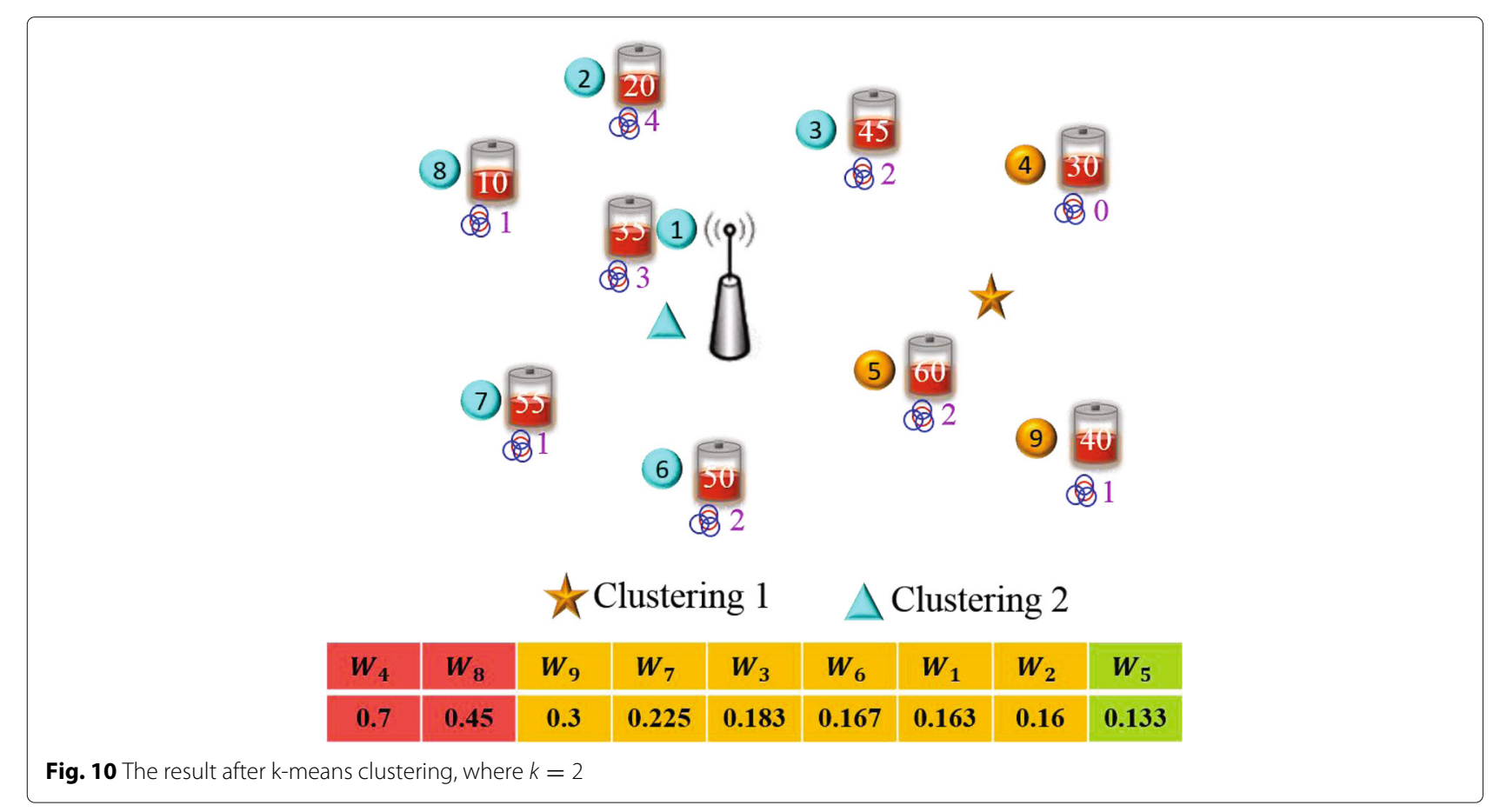

out of energy and coverage holes occurs. On the contrary, PERS and e-PERS proposed in the paper do not result in a coverage hole until the end of the simulation. It reveals that the proposed schemes, PERS and e-PERS, can effectively avoid the occurrence of coverage hole and sustain the network lifetime.

Figure 12 illustrates the remaining energy of sensors at the end of the simulation, where the blue lines stand for the PERS and the red ones for the e-PERS. Obviously, no sensor depletes its energy at the end of the simulation, no matter the replenishment scheme is PERS or e-PERS. Moreover, we can see that e-PERS maintains the effectiveness of power better than PERS, because e-PERS clusters sensors and the mobile charger can have an efficient charging tour than that of PERS. This can effectively reduce the energy consumption.

Table 2 Simulation setting

\begin{tabular}{ll}
\hline Parameters & Values \\
\hline Area size & $300 \mathrm{~m} \times 300 \mathrm{~m}$ \\
Number of sensors & 80 \\
Capacity of battery $(B)$ & $500 \mathrm{~J}$ \\
Capacity of mobile charger $(C)$ & $2000 \mathrm{~J}$ \\
Crun & $100 \mathrm{~J} / \mathrm{s}$ \\
Speed of mobile charger & $5 \mathrm{~m} / \mathrm{s}$ \\
Charging rate & $5 \mathrm{~J} / \mathrm{s}$ \\
\hline
\end{tabular}

\section{Conclusions}

In the past research, the authors considered the remaining energy of sensors as the only clue to recharge sensors. However, it is obvious that only consider the remaining energy of sensors is not enough to avoid the occurrences of coverage holes. As a result, this paper not only considers the remaining energy of sensors but also takes the coverage degree of sensors in terms of cover sets into consideration. We proposed PERS and e-PERS, two efficient recharging schemes, in the paper. The two schemes can make the energy replenishment of sensors more efficiently and avoid charging the sensors with a lower coverage degree to maintain the coverage of the network. In the future, multiple mobile chargers will be considered and can cooperate and coordinate to enhance the efficiency of the recharging of sensors.

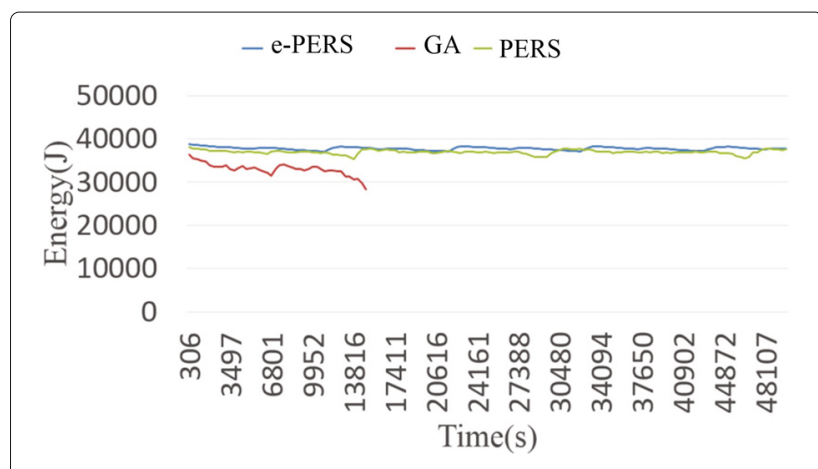

Fig. 11 Performance comparison in terms of network lifetime 


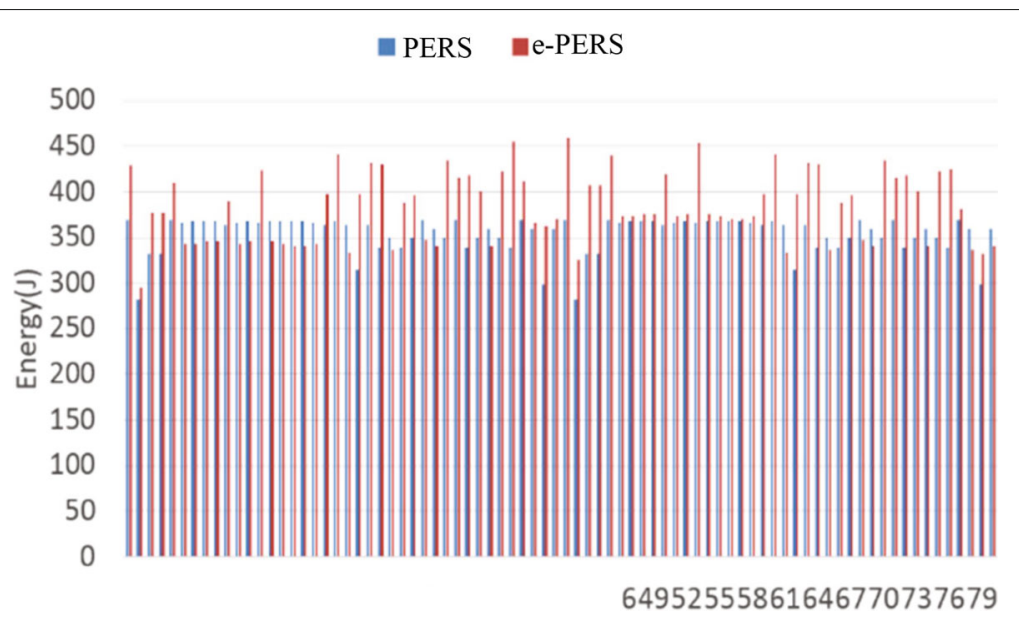

Fig. 12 The remaining energy of sensors at the end of the simulation

\section{Funding}

The work was partially supported by the Ministry of Science and Technology of the Republic of China under Grant No. MOST 106-2221-E-032-004.

\section{Authors' contributions}

The study conception and design of the paper, drafting of the manuscript, and critical revision are contributed by the first author. The acquisition of data and analysis and interpretation of data are contributed by the second author. Both authors read and approved the final manuscript.

\section{Competing interests}

The authors declare that they have no competing interests.

\section{Publisher's Note}

Springer Nature remains neutral with regard to jurisdictional claims in published maps and institutional affiliations.

Received: 30 July 2017 Accepted: 5 December 2017

Published online: 19 December 2017

\section{References}

1. IF Akyildiz, W Su, Y Sankarasubramaniam, E Cayirci, Wireless sensor networks: a survey. Comput. Netw. 38, 393-422 (2002)

2. LML Oliveira, JJP Rodrigues, Wireless sensor networks: a survey on environmental monitoring. J. Commun. 6(2), 143-151 (2011)

3. P Rawat, KD Singh, H Chaouchi, JM Bonnin, Wireless sensor networks: a survey on recent developments and potential synergies. J. Supercomput. 68, 1-48 (2014)

4. J Yick, B Mukherjee, D Ghosal, Wireless sensor networks survey. Comput. Netw. 52, 2292-2330 (2008)

5. B Carbunar, A Grama, J Vitek, O Carbunar, in Proceedings of the IEEE Communications Society Conference on Sensors, Mesh and Ad Hoc Communications and Networks (SECON). Coverage preserving redundancy elimination in sensor networks, (2004), pp. 377-386

6. H Gupta, SR Das, Q Gu, in Proceedings of the ACM International Symposium on Mobile Ad Hoc Networking and Computing (MOBIHOC). Connected sensor cover: self-organization of sensor networks for efficient query execution, (2003), pp. 189-200

7. KP Shih, YD Chen, CW Chiang, BC Liu, in Proceedings of the Workshop on Wireless, Ad Hoc, and Sensor Networks (WASN). Geo-BASS: distributed geometric-based activity scheduling schemes for wireless sensor networks, (2005)

8. $\mathrm{F} Y e, \mathrm{G}$ Zhong, J Cheng, S Lu, L Zhong, in Proceedings of the International Conference on Distributed Computing Systems (ICDCS). PEAS: a robust energy conserving protocol for long-lived sensor network, (2003), pp. 28-37
9. A Kurs, A Karalis, R Moffatt, JD Joannopoulos, P Fisher, M Soljacic, Wireless power transfer via strongly coupled magnetic resonances. Science. $\mathbf{3 8}$, 83-86 (2007)

10. KW Fan, Z Zheng, P Sinha, in Proceedings of the ACM Conference on Embedded Network Sensor Systems (SenSys). Steady and fair rate allocation for rechargeable sensors in perpetual sensor networks, (2008), pp. 239-252

11. W Liang, $X$ Ren, $X$ Jia, $X X$ u, Monitoring quality maximization through fair rate allocation in harvesting sensor networks. IEEE Trans Parallel Distrib. Syst. 24(9), 1827-1840 (2013)

12. CM Vigorito, D Ganesan, AG Barto, in Proceedings of the IEEE Communications Society Conference on Sensors, Mesh and Ad Hoc Communications and Networks (SECON). Adaptive control of duty cycling in energy-harvesting wireless sensor networks, (2007), pp. 21-30

13. KXiong, P Fan, C Zhang, KB Letaief, Wireless information and energy transfer for two-hop non-regenerative MIMO-OFDM relay networks. IEEE J. Sel. Areas Commun. 33(8), 1595-1611 (2015)

14. J Liu, K Xiong, P Fan, Z Zhong, RF energy harvesting wireless powered sensor networks for smart cities. IEEE Access. 5, 9348-9358 (2017)

15. KXiong, C Chen, G Qu, P Fan, KB Letaief, Group communication with optimal resource allocation in wireless powered communication networks. IEEE Trans. Wirel. Commun. 16(6), 3840-3853 (2017)

16. S Guo, C Wang, Y Yang, in Proceedings of the IEEE INFOCOM, the Annual Joint Conference of the IEEE Computer and Communications Societies. Mobile data gathering with wireless energy replenishment in rechargeable sensor networks, (2013), pp. 1932-1940

17. L He, P Cheng, Y Gu, J Pan, T Zhu, C Liu, in Proceedings of the IEEE INFOCOM, the Annual Joint Conference of the IEEE Computer and Communications Societies. Mobile-to-mobile energy replenishment in mission-critical robotic sensor networks, (2014), pp. 1195-1203

18. L He, L Kong, Y Gu, J Pan, T Zhu, Evaluating the on-demand mobile charging in wireless sensor networks. IEEE Trans. Mob. Comput. 14(9), 1861-1875 (2015)

19. K Li, H Luan, CC Shen, in Proceedings of the IEEE Wireless Communications and Networking Conference (WCNC). Qi-ferry: energy-constrained wireless charging in wireless sensor networks, (2012), pp. 2515-2520

20. X Ren, W Liang, W Xu, in Proceedings of the International Conference on Computer Communication and Networks (ICCCN). Maximizing charging throughput in rechargeable sensor networks, (2014), pp. 1-8

21. Y Shi, L Xie, YT Hou, HD Sherali, in Proceedings of the IEEE INFOCOM, the Annual Joint Conference of the IEEE Computer and Communications Societies. On renewable sensor networks with wireless energy transfer, (2011), pp. 1350-1358

22. C Wang, Y Yang, J Li, in Proceedings of the IEEE Wireless Communications and Networking Conference (WCNC). Stochastic mobile energy replenishment and adaptive sensor activation for perpetual wireless rechargeable sensor networks, (2013), pp. 974-979 
23. CWang, J Li, F Ye, Y Yang, in Proceedings of the IEEE Communications Society Conference on Sensors, Mesh and Ad Hoc Communications and Networks (SECON). Recharging schedules for wireless sensor networks with vehicle movement costs and capacity constraints, (2014), pp. 468-476

24. C Wang, J Li, F Ye, Y Yang, NETWRAP: An NDN based real-time wireless recharging framework for wireless sensor networks. IEEE Trans. Mob. Comput. 13(6), 1283-1297 (2014)

25. L Xie, Y Shi, YT Hou, W Lou, HD Sherali, SF Midkiff, in Proceedings of the IEEE Communications Society Conference on Sensors, Mesh and Ad HoC Communications and Networks (SECON). On renewable sensor networks with wireless energy transfer: the multi-node case, (2012), pp. 10-18

26. M Zhao, J Li, Y Yang, A framework of joint mobile energy replenishment and data gathering in wireless rechargeable sensor networks. IEEE Trans. Mob. Comput. 13(12), 2689-2705 (2014)

27. SY Wang, KP Shih, YD Chen, HH Ku, in Proceedings of the IEEE Wireless Communications and Networking Conference (WCNC). Preserving target area coverage in wireless sensor networks by using computational geometry, (2010)

28. CF Huang, YC Tseng, The coverage problem in a wireless sensor network. ACM Mob. Netw. Appl. (MONET). 10(4), 519-528 (2005)

29. THe, C Huang, BM Blum, JA Stankovic, T Abdelzaher, in Proceedings of the ACM International Conference on Mobile Computing and Networking (MOBICOM). Range-free localization schemes for large scale sensor networks, (2003), pp. 81-95

30. $\mathrm{X} \mathrm{Ji}, \mathrm{H}$ Zha, in Proceedings of the IEEE INFOCOM, the Annual Joint Conference of the IEEE Computer and Communications Societies. Sensor positioning in wireless ad-hoc sensor networks using multidimensional scaling, (2004)

31. TH Cormen, CE Leiserson, RL Rivest, C Stein, Introduction to Algorithms, 3rd edn. (The MIT Press, 2009)

\section{Submit your manuscript to a SpringerOpen ${ }^{\circ}$ journal and benefit from:}

- Convenient online submission

- Rigorous peer review

- Open access: articles freely available online

- High visibility within the field

- Retaining the copyright to your article

Submit your next manuscript at $>$ springeropen.com 